\title{
Oil/water Microreactor with Core-shell Wetting State on a SOB/OL-SHB/HL Multilevel Patterned Surface
}

\author{
Long Jiao ${ }^{\mathrm{a}, \mathrm{b}, \mathrm{c}, \mathrm{e}, *}$, Yixiao $\mathrm{Wu}^{\mathrm{a}, \mathrm{b}, *}$, Yanjun $\mathrm{Hu}^{\mathrm{a}, \mathrm{b}}$, Huaping $\mathrm{Wu}^{\mathrm{a}}$, \\ Zhang Xu $u^{\mathrm{a}, \mathrm{b}}$, Long Han ${ }^{\mathrm{a}, \mathrm{b}}$, Qianqian Guo ${ }^{\mathrm{a}, \mathrm{b}}$, Dongliang $\mathrm{Li}^{\mathrm{d}}$, Rong Chen ${ }^{\mathrm{d}}$ \\ ${ }^{a}$ College of Mechanical Engineering, Zhejiang University of Technology, Hangzhou 310023, \\ China \\ ${ }^{b}$ Institute of Energy and Power Engineering, Zhejiang University of Technology, Hangzhou \\ 310023, China \\ ${ }^{c}$ Key Laboratory of E\&M (Zhejiang University of Technology), Ministry of Education \& \\ Zhejiang Province, Hangzhou 310023, China \\ ${ }^{\mathrm{d}}$ Key Laboratory of Low-grade Energy Utilization Technologies and Systems (Chongqing \\ University), Ministry of Education, Chongqing 400030, China \\ e Zhejiang Tuff Development Co., Ltd., Jiaxing 314400, China \\ *Corresponding author. E-mail: jiaolong@zjut.edu.cn (Long Jiao), wuyixiao513@163.com \\ (Yixiao $\mathrm{Wu}$ )
}




\section{Supplementary figures}

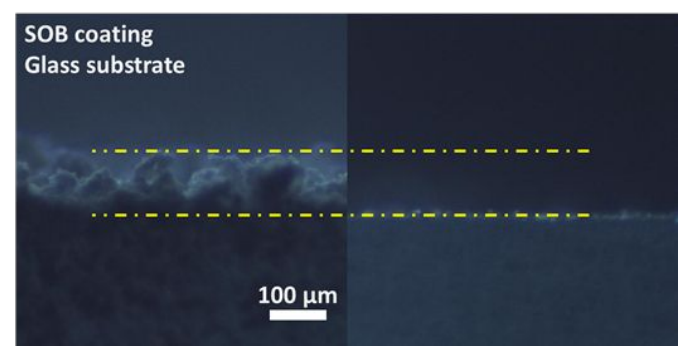

a

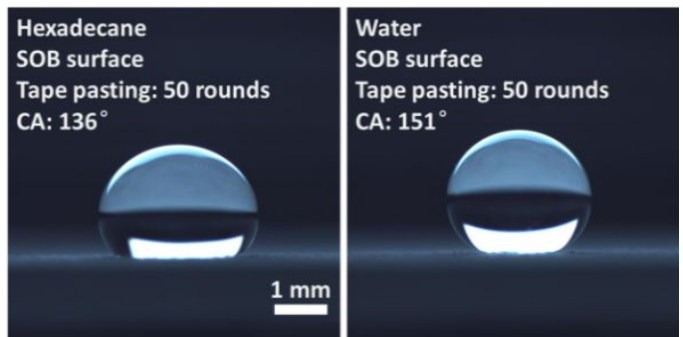

C

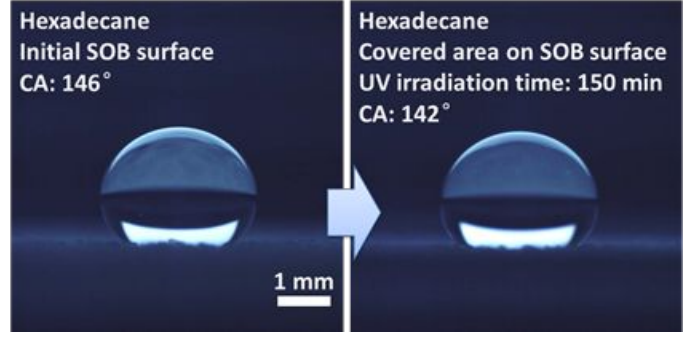

e

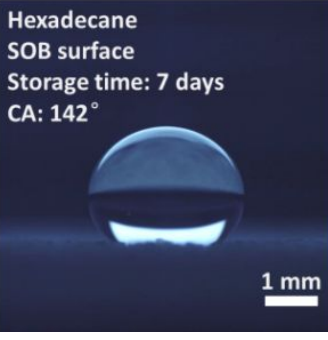

Water

SOB surface

Storage time: 7 days

$\mathrm{CA}: 147^{\circ}$

CA: $147^{\circ}$

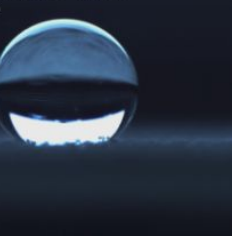

b

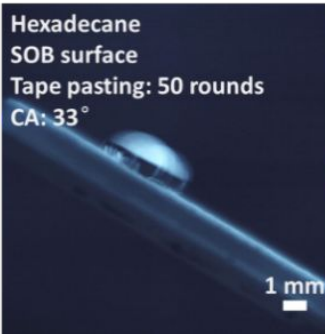

\section{Water}

SOB surface

Tape pasting: 50 rounds

SA: 16

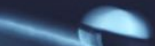

$\mathrm{mm}$

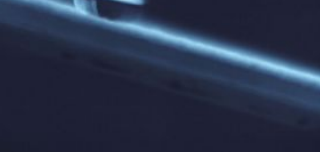

d

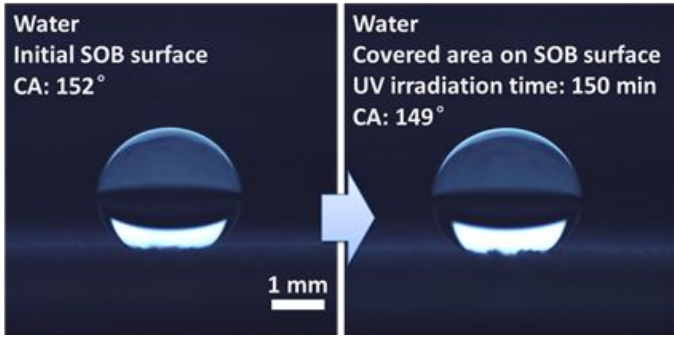

f

Fig. S1. (a) SOB coating thickness. (b) Interface morphologies of hexadecane and water droplets on the SOB surface after a storage time of 7 days. (c) Interface morphologies and (d) slides of hexadecane and water droplets on the SOB surface with tape pasting number of 50. Interface morphologies of (e) hexadecane and (f) water on the covered area on the SOB surface before and after UV exposure for 150 min. The droplet volume equaled $10 \mu \mathrm{L}$. 


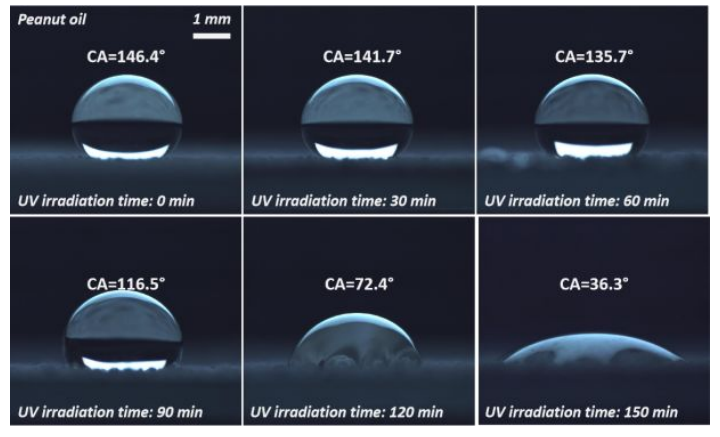

a

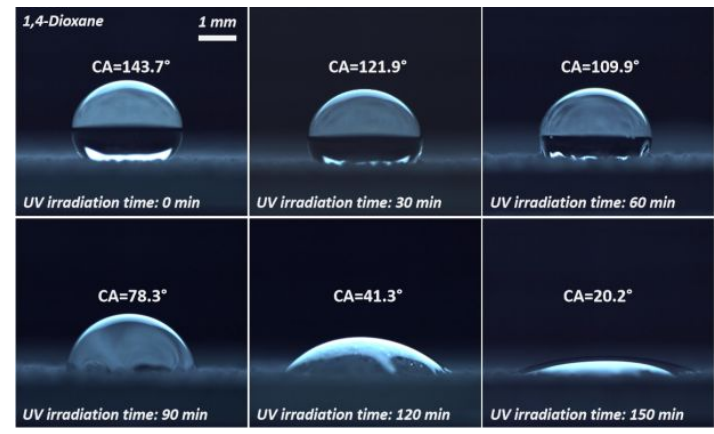

$\mathrm{b}$
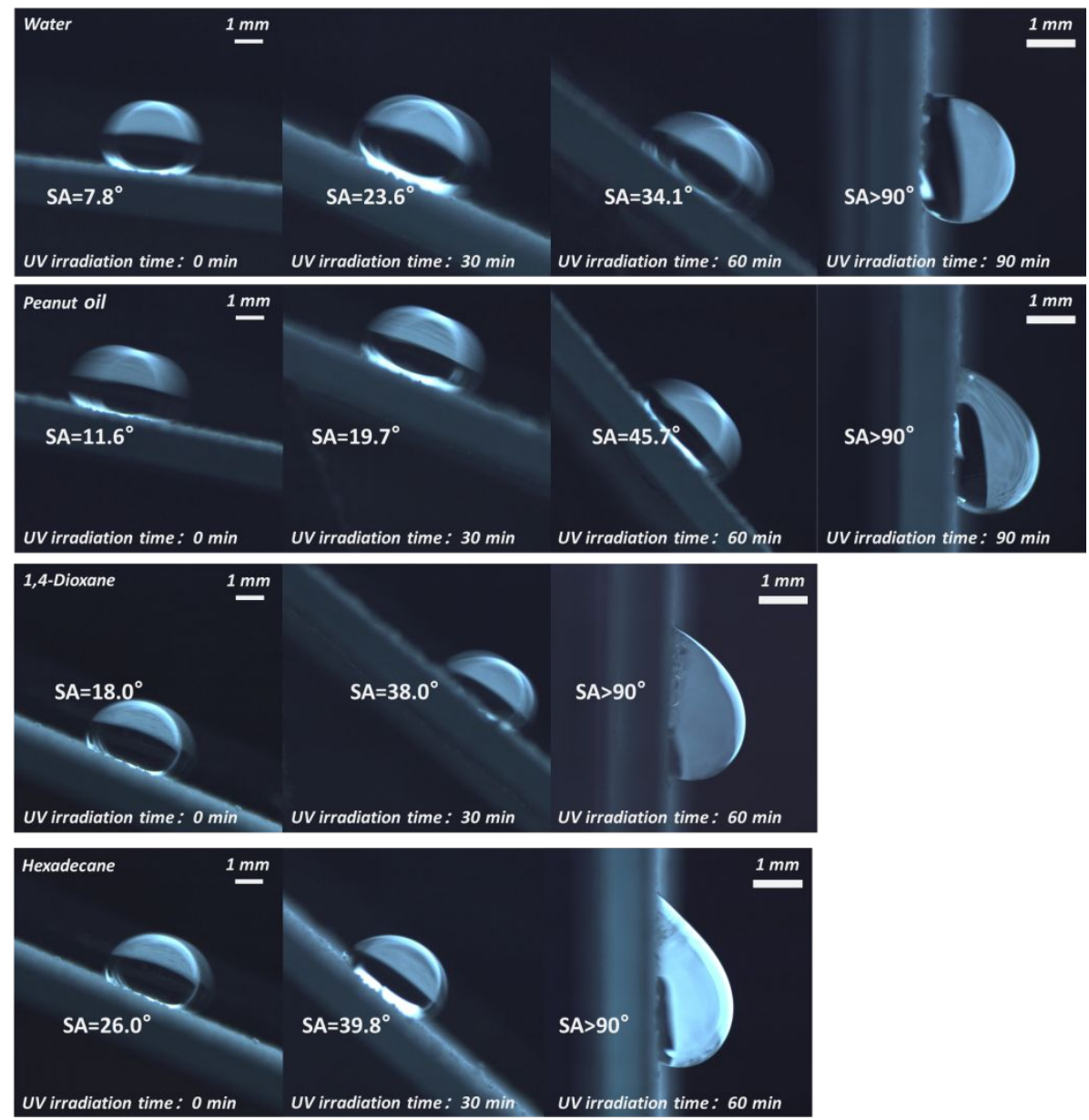

c

Fig. S2. Interface morphologies of (a) peanut oil and (b) 1,4-dioxane droplets on UV-modified homogeneous surfaces with different UV irradiation times. (c) SAs of water, peanut oil, 1,4-dioxane, hexadecane droplets on UV-modified homogeneous surfaces with different UV irradiation times. The droplet volume was $10 \mu \mathrm{L}$. 

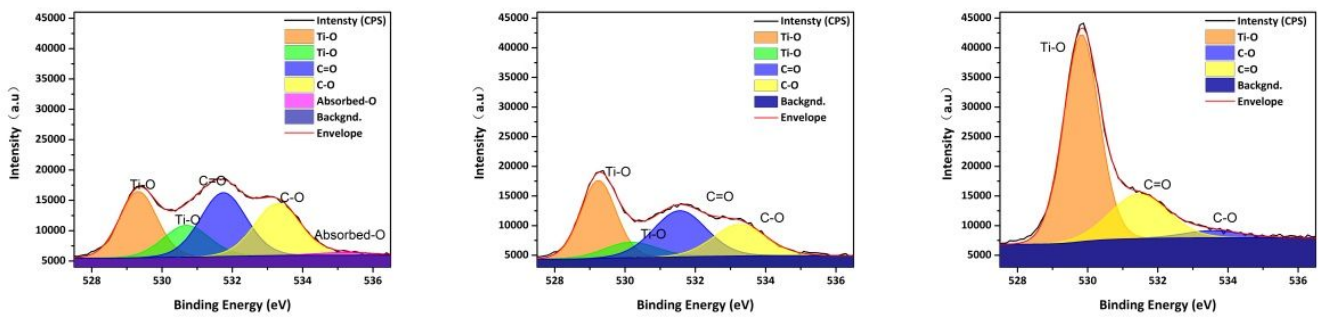

Fig. S3. High-resolution XPS images of O1 s of SOB background, OL-SHB area, and HL area on SOB/OL-SHB/HL surface. 


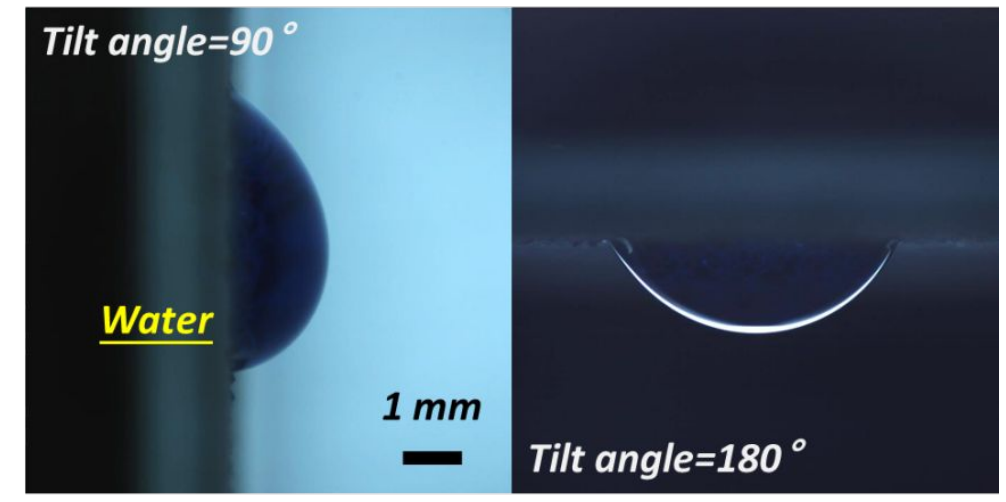

a

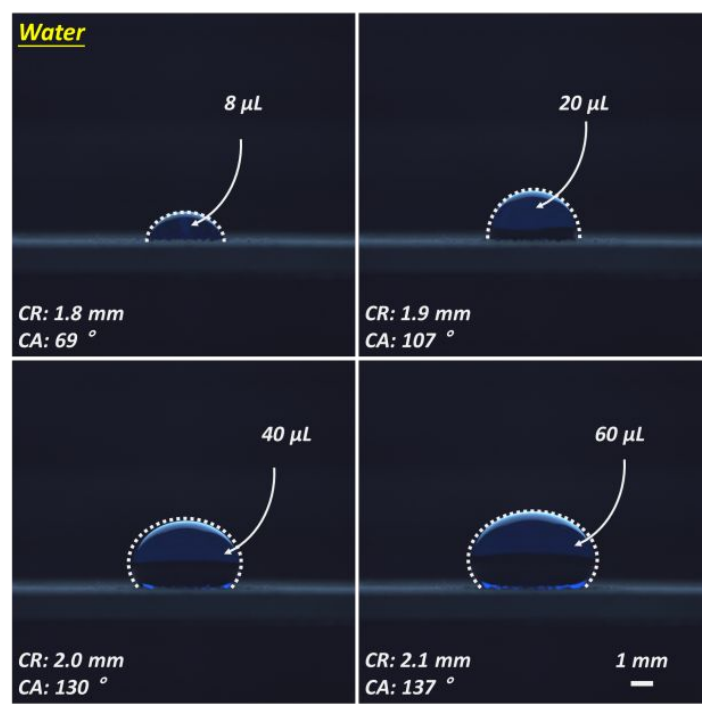

$\mathrm{b}$
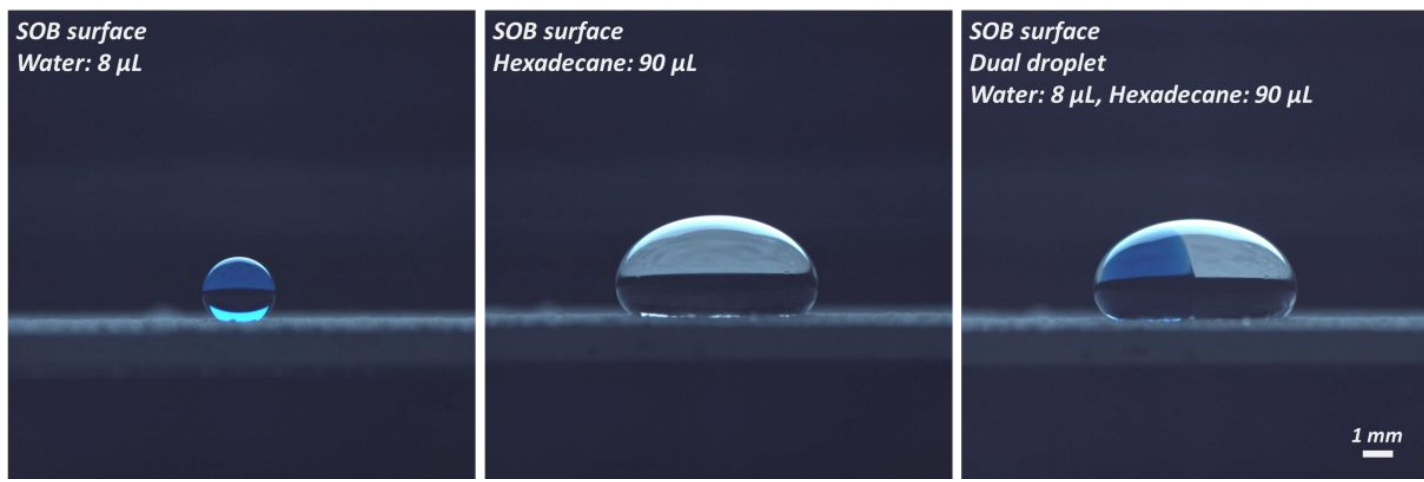

d

Fig. S4. (a) Adhesion of water droplets on the SOB/OL-SHB/HL surface. (b) Interface morphologies of water droplets with different volumes on the SOB/OL-SHB/HL surface. (c) The slide and split of the oil/water microreactor when tilting the SOB/OL-SHB/HL surface to a certain angle. (d) Interface morphologies of 
water, hexadecane, and water-hexadecane droplets on the SOB surface. The droplet volume was $10 \mu \mathrm{L}$ in (a). 


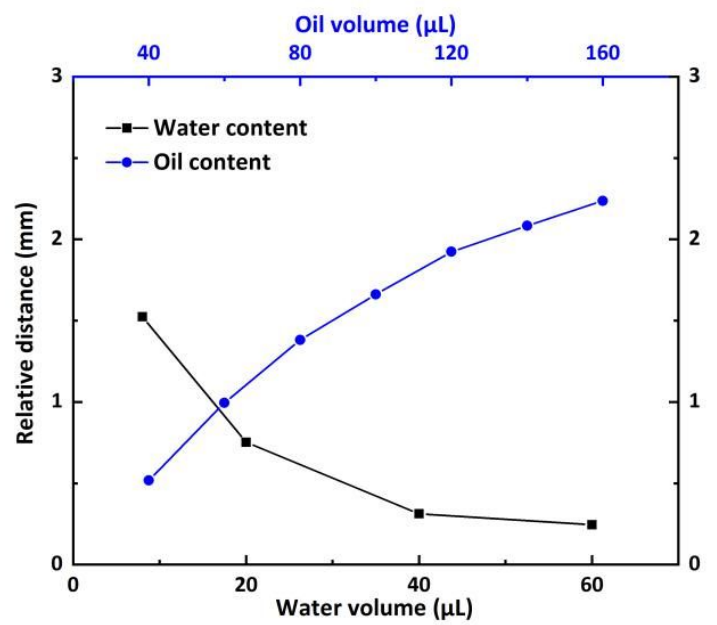

Fig. S5. Influence of oil and water volumes on relative distance equaling that total height of oil/water microreactor minus initial individual water droplet height. 


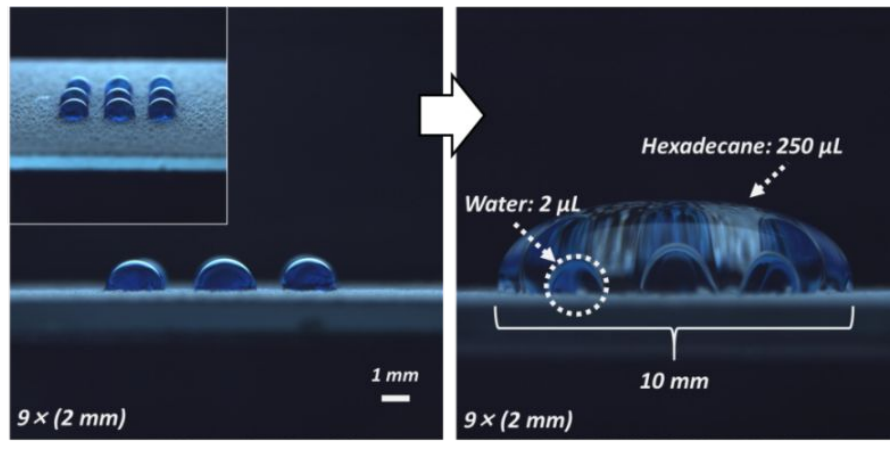

a
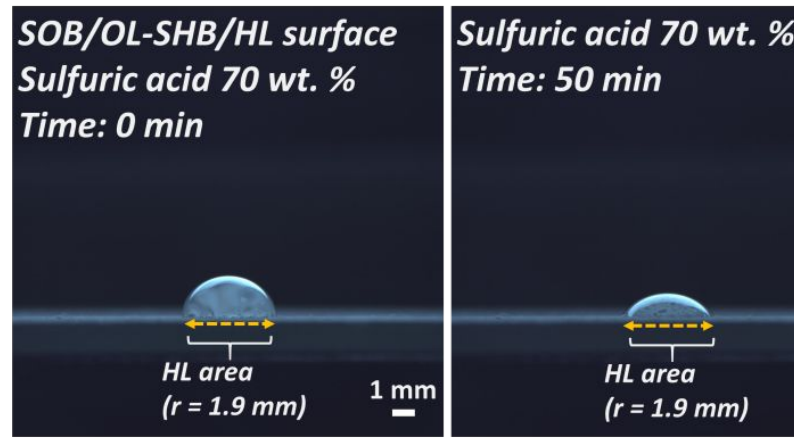
Time: $\mathbf{5 0}$ min

Undamaged surface

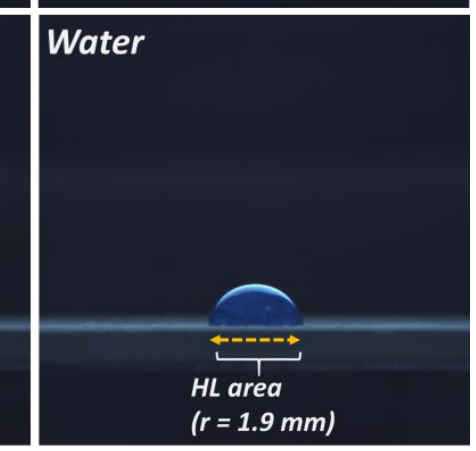

$\mathrm{b}$

Fig. S6. (a) Images of an oil/water microreactor with multiple inner son droplets. (b) Durability of the SOB/OL-SHB/HL surface for a highly corrosive droplet of $70 \mathrm{wt}$ \% sulfuric acid 


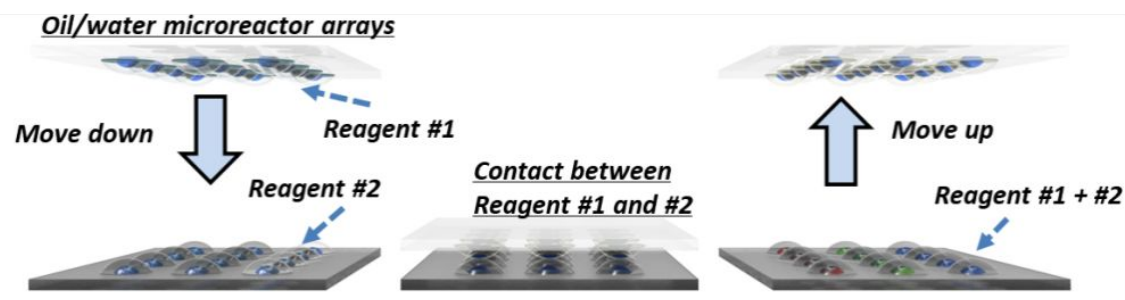

a $\quad$ b

c
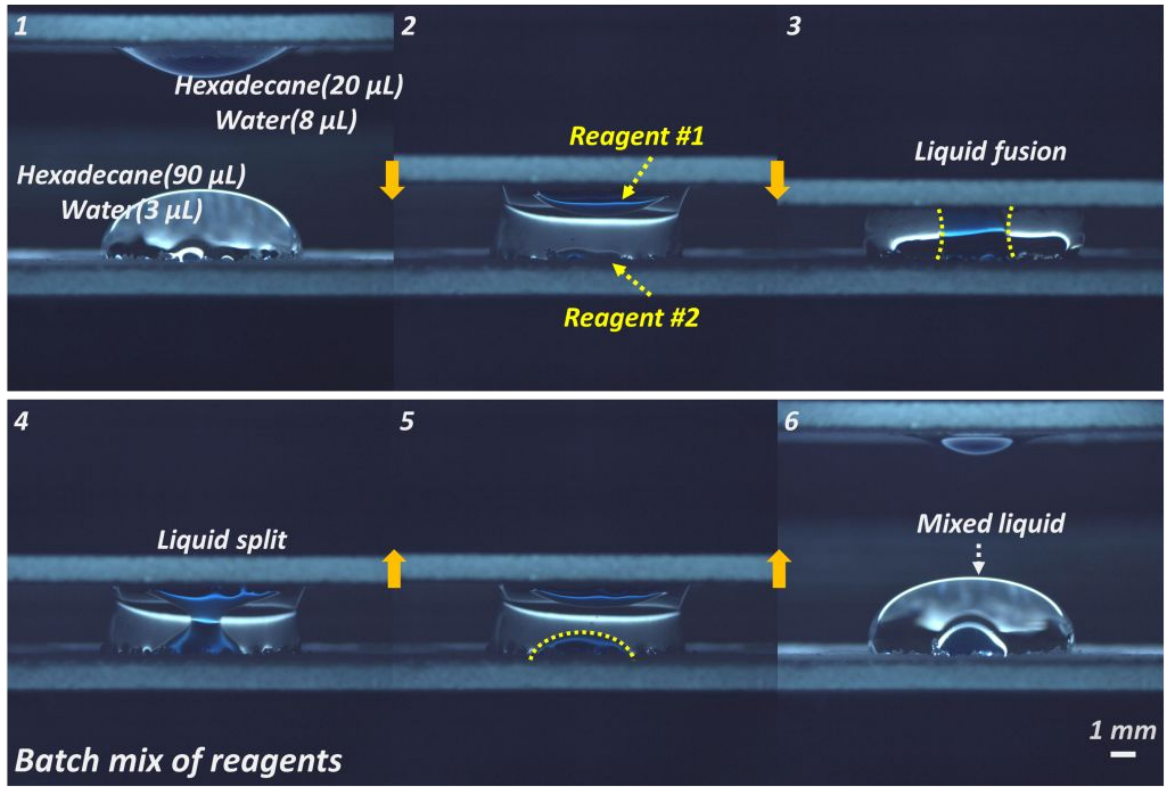

d

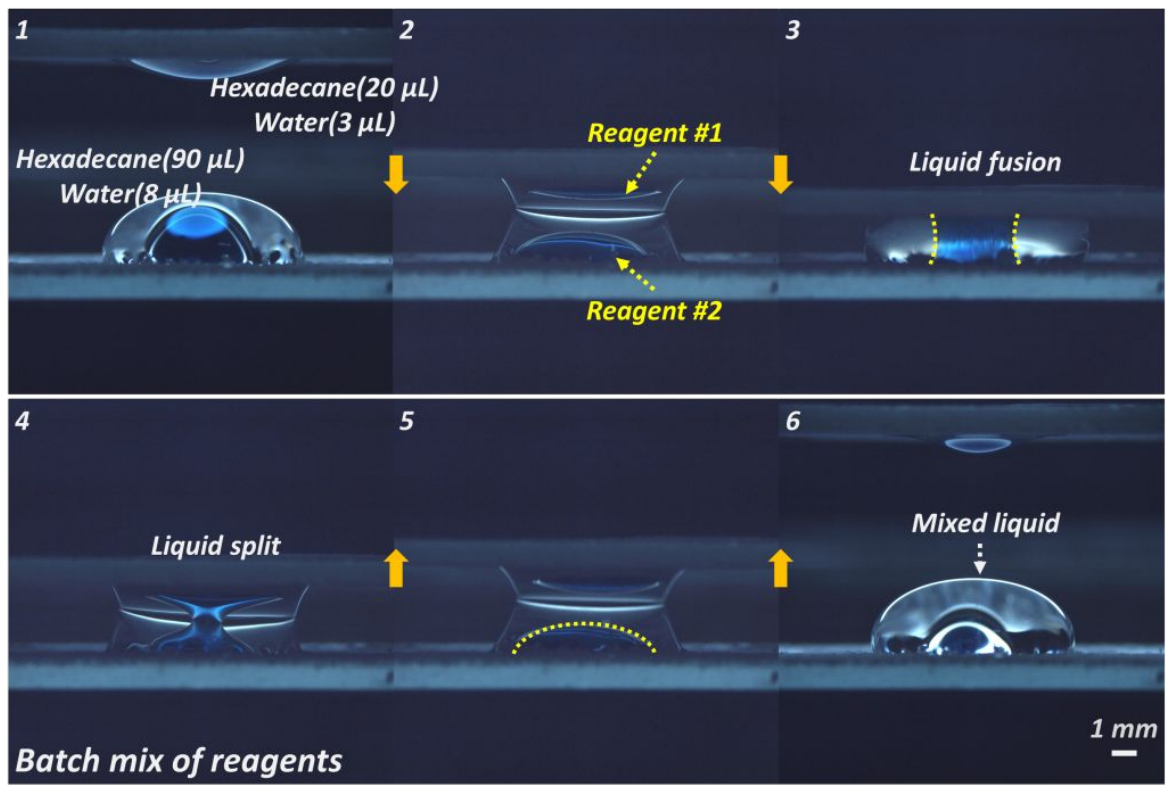

e

Fig. S7. Batch mix of reagents obtained by sandwiching two oil/water microreactor arrays. (a) Gradually closing two oil/water microreactor arrays with different core 
liquids (Reagent \#1 and Reagent \#2). (b) Contact of droplet cores in a pair of oil/water microreactors. (c) Generating two new oil/water microreactor arrays with a mixed droplet core (Reagent \#1+\#2). (d) Images of the mixing and splitting processes of a pair of oil/water microreactors with Reagent \#1/Reagent \#2 ratios of (d) 3/8 and (e) $8 / 3$.

\section{Illustration for Fig. S7.}

The batch mix and split of the oil/water microreactors could be achieved by sandwiching two arrays. As shown in Fig. S7a, an oil/water microreactor array with Reagent \#1 was upside down, while another oil/water microreactor array with Reagent \#2 was placed normally. When moving the upper array down, each pair of oil/water microreactors came into contact, resulting in the fusion of the oil packing layers and the water droplet cores. Reagent \#1 and Reagent \#2 were mixed, as shown in Fig. S7b. Then, the two substrates were separated, generating two new oil/water microreactor arrays with the mixed liquid core (Reagent \#1-\#2), as shown in Fig. S7c. These processes are shown in Fig. S7d, S7e, which indicated that the initial droplet core volumes could change the mixing ratio. As shown in Fig. S7d and S7e, Reagent $\# 1 /$ Reagent \#2 ratios of 3/8 and 8/3 were realized. 


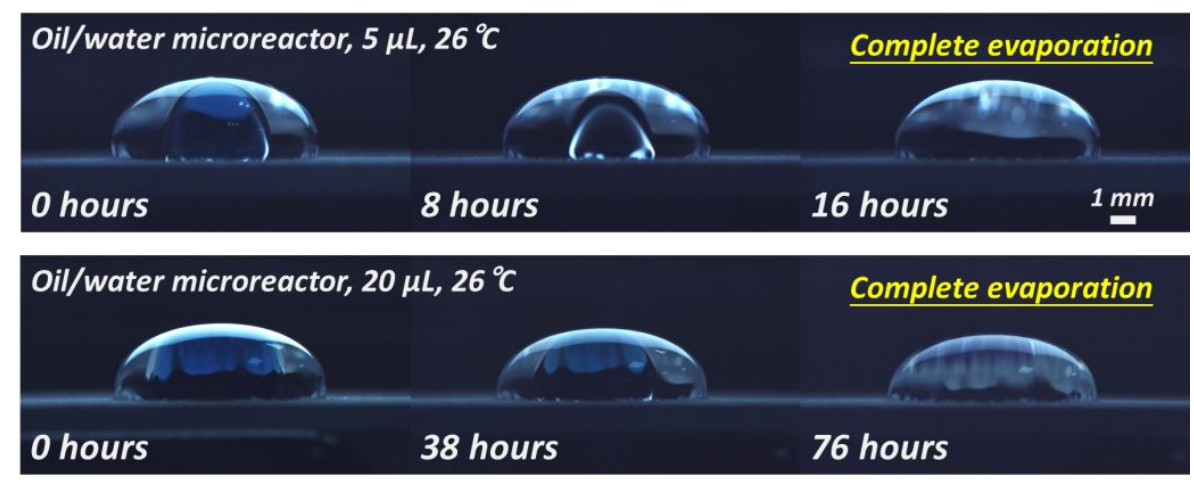

a

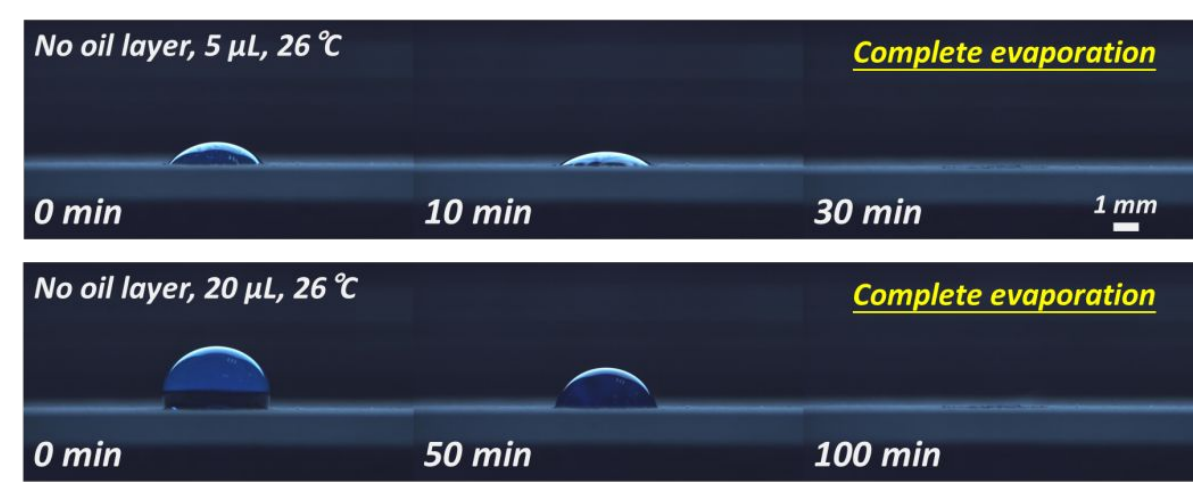

$\mathrm{b}$

Fig. S8. Evaporation processes of water droplets with different initial masses (a) with and (b) without a hexadecane packing layer at $26^{\circ} \mathrm{C}$. 


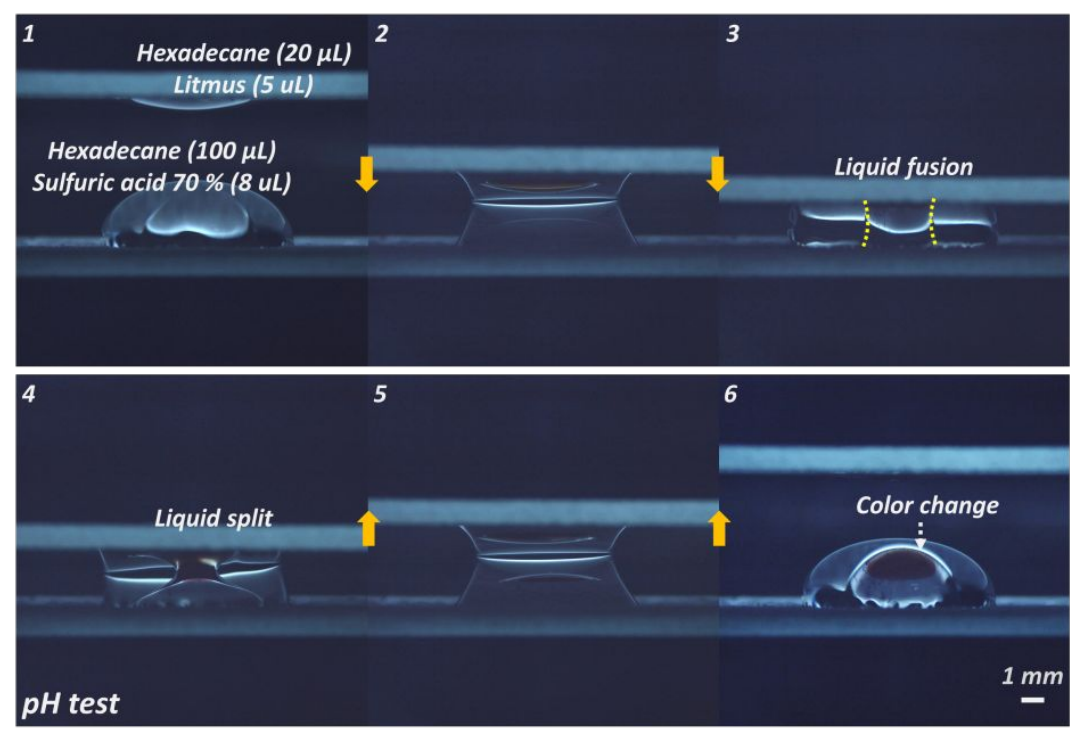

a
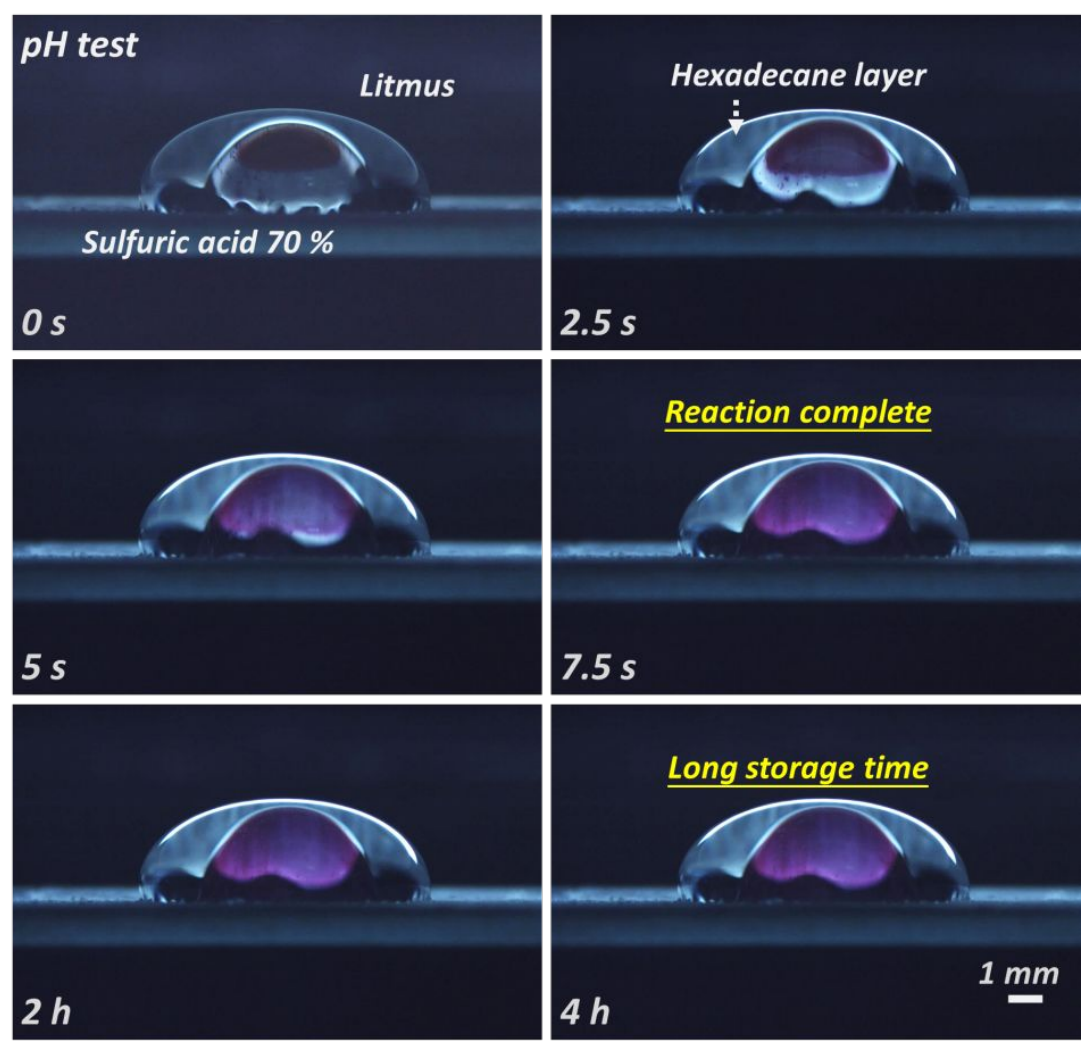

$\mathrm{b}$

Fig. S9. pH test using an oil/water microreactor on a SOB/OL-SHB/HL surface. (a) Images of the mixing and splitting processes of a pair of corresponding oil/water microreactors with litmus and 70\% sulfuric acid. (b) Oil/water microreactor-based $\mathrm{pH}$ indicator with a rapid response time and a long storage time. 


\begin{tabular}{|c|c|c|c|c|}
\hline PEAK & $\begin{array}{c}\text { Energy } \\
(\mathrm{eV})\end{array}$ & $\begin{array}{c}\text { Chemical } \\
\text { state }\end{array}$ & $\begin{array}{c}\text { Atomic } \\
\text { Concentration } \\
(\%)\end{array}$ & $\begin{array}{c}\text { Aggregate } \\
\text { Atomic } \\
\text { Concentration } \\
(\%)\end{array}$ \\
\hline C1 s & 284.76 & C-C/C-H & 17.51 & \multirow{6}{*}{46.98} \\
\hline C1 s & 286.2 & $\mathrm{C}-\mathrm{N} / \mathrm{C}-\mathrm{O}$ & 9.78 & \\
\hline C1 s & 288.6 & $\mathbf{C}=\mathbf{O}$ & 4.52 & \\
\hline C1 s & 290.7 & $C^{*}$ F2-CHx & 4.02 & \\
\hline C1 s & 291.49 & $C * F 2-C F x$ & 8.40 & \\
\hline C1 s & 293.65 & CF3 & 2.75 & \\
\hline $01 \mathrm{~s}$ & 529.33 & Ti-O & 3.88 & \multirow{5}{*}{14.72} \\
\hline $01 \mathrm{~s}$ & 530.7 & Ti-O & 2.28 & \\
\hline $01 \mathrm{~s}$ & 531.74 & $\mathbf{C}=\mathbf{O}$ & 4.58 & \\
\hline $01 \mathrm{~s}$ & 533.29 & $\mathrm{C}-\mathrm{O}$ & 3.74 & \\
\hline O1 s & 535.24 & Absorbed-O & 0.24 & \\
\hline F1 s & 689.08 & organic-F & 38.29 & 38.29 \\
\hline
\end{tabular}




\begin{tabular}{|c|c|c|c|c|}
\hline PEAK & $\begin{array}{c}\text { Energy } \\
(\mathrm{eV})\end{array}$ & $\begin{array}{c}\text { Chemical } \\
\text { state }\end{array}$ & $\begin{array}{c}\text { Atomic } \\
\text { Concentration } \\
(\%)\end{array}$ & $\begin{array}{c}\text { Aggregate } \\
\text { Atomic } \\
\text { Concentration } \\
(\%)\end{array}$ \\
\hline C1 s & 284.81 & C-C/C-H & 18.79 & \multirow{6}{*}{45.84} \\
\hline C1 s & 286.38 & C-N/C-O & 8.44 & \\
\hline C1s & 288.68 & $\mathbf{C}=\mathbf{O}$ & 4.74 & \\
\hline C1 s & 290.77 & $C^{*}$ F2-CHx & 4.13 & \\
\hline C1 s & 291.54 & $C^{*} \mathbf{F} 2-C F x$ & 7.41 & \\
\hline C1 s & 293.59 & CF3 & 2.33 & \\
\hline $01 \mathrm{~s}$ & 529.24 & Ti-O & 6.93 & \multirow{4}{*}{19.42} \\
\hline $01 \mathrm{~s}$ & 530.17 & Ti-O & 2.06 & \\
\hline $01 \mathrm{~s}$ & 531.56 & $\mathbf{C}=\mathbf{O}$ & 6.2 & \\
\hline $01 \mathrm{~s}$ & 533.26 & $\mathrm{C}-\mathrm{O}$ & 4.23 & \\
\hline F1 s & 688.46 & organic-F & 34.73 & 34.74 \\
\hline
\end{tabular}

b 


\begin{tabular}{|c|c|c|c|c|}
\hline PEAK & $\begin{array}{c}\text { Energy } \\
(\mathrm{eV})\end{array}$ & $\begin{array}{c}\text { Chemical } \\
\text { state }\end{array}$ & $\begin{array}{c}\text { Atomic } \\
\text { Concentration } \\
(\%)\end{array}$ & $\begin{array}{c}\text { Aggregate } \\
\text { Atomic } \\
\text { Concentration } \\
(\%)\end{array}$ \\
\hline C1 s & 284.71 & $\mathrm{C}-\mathrm{C} / \mathrm{C}-\mathrm{H}$ & 17.63 & \multirow{6}{*}{35.87} \\
\hline C1s & 286.36 & $\mathrm{C}-\mathrm{N} / \mathrm{C}-\mathrm{O}$ & 9.09 & \\
\hline C1 s & 288.91 & $\mathbf{C}=\mathbf{O}$ & 3.79 & \\
\hline C1 s & 291.29 & C*F2-CHx & 1.16 & \\
\hline C1 s & 292.04 & C*F2-CFx & 3.20 & \\
\hline C1s & 294.15 & CF3 & 1.00 & \\
\hline O1 s & 529.82 & Ti-O & 34.60 & \multirow{3}{*}{48.57} \\
\hline $01 \mathrm{~s}$ & 531.46 & $\mathbf{C}=\mathbf{O}$ & 12.05 & \\
\hline O1 s & 533.56 & C-O & 1.92 & \\
\hline F1s & 684.03 & Fluoride (Ti-F) & 0.91 & \multirow{3}{*}{15.56} \\
\hline F1 s & 687.36 & Ti-F-O & 0.71 & \\
\hline F1 s & 689.05 & organic-F & 13.95 & \\
\hline
\end{tabular}

Table S1. Chemical states and atomic concentrations of C, O, and F elements on the (a) SOB background, (b) OL-SHB area, and (c) HL area on the SOB/OL-SHB/HL surface. 